\title{
Vertically Aligned Hybrid Core/Shell Semiconductor Nanowires for Photonics Applications
}

\author{
Manuel Macias-Montero, A. Nicolas Filippin, Zineb Saghi, Francisco J. Aparicio, \\ Angel Barranco, Juan P. Espinos, Fabian Frutos, Agustin R. Gonzalez-Elipe, \\ and Ana Borras*
}

\begin{abstract}
A family of 1D organic/inorganic core/shell materials formed by an inner organic nanowire (ONW) conformally covered with an inorganic wide band gap semiconductor $\left(\mathrm{ZnO}\right.$ or $\left.\mathrm{TiO}_{2}\right)$ layer is presented. The developed procedure is a two-steps vacuum methodology involving the formation of supported single crystal small-molecule nanowires by physical vapor deposition and plasma enhance chemical vapor deposition (PECVD) of the inorganic shell. Critical characteristics of the last technique are the possibilities of low temperature and remote configuration deposition. Additionally, an initial step has to be included in order to create nucleation centers for the growth of the ONWs. The procedure and its general character in terms of the variability in organic core and inorganic shells composition and the applicability of the technique to different substrates are presented. The formation of the inorganic shell with no damage of the organic core single-crystalline structure is demonstrated by high resolution transmission electron microscopy. The vertical alignment of the hybrid nanostructure is achieved thanks to the interaction of the 1D organic nanostructured surfaces and the glow discharge during the deposition of the inorganic shell by PECVD. The optical properties of these core/shell NWs are studied by fluorescence spectroscopy and microscopy, and their application as nanoscale waveguides in the 550-750 $\mathrm{nm}$ range addressed.
\end{abstract}

\section{Introduction}

The synthesis of one-dimensional nanostructures has attracted an enormous attention in the last decade because of their outstanding shape (chemistry, size, and material) dependent properties. ${ }^{[1]}$ An important step forward in the field is the growth of 1D heterostructures by, for example, synthesizing two-phase heterojunctions between metals, semiconductors, carbon, and polymers. ${ }^{[1,2]}$ Simultaneously, the controlled fabrication of core/ shell 1D nanostructures has emerged as an important topic in diverse fields. ${ }^{[3]}$ For instance, combination of different metals, metal/metal-oxide, oxide/oxide NWs, and nanorods has been intended for high-performance supercapacitors, solar cells, self-cleaning surfaces, and photonic materials. ${ }^{[4 a-e]}$ Lately, organic/ inorganic and inorganic/organic combinations have been proposed as high capacity anode for lithium batteries ${ }^{[4 f]}$ and photocatalysis. ${ }^{[4 \mathrm{~g}]}$ In this context, recent molecular electronic developments have promoted the fabrication of optic and electronic devices ${ }^{[5]}$ based on organic molecules, 1D organic nanostructures and, to a much lesser extent, 1D organic heterostructures. ${ }^{[6]}$ The synthesis and processing of organic nanowires (ONWs) from $\pi$-conjugated small-molecules have been accomplished by several methodologies involving the use of inorganic and organic templates, liquid and surface crystallization processes, or vapor transport methods. ${ }^{[5]}$ The latter ones ${ }^{[5,6 a-c, e-f, 7]}$ provide a strict control on the composition, crystal structure, and morphology of the ONWs. In particular, it has been already shown that the vapor transport method at low pressure or physical vapor deposition (PVD) represents a facile and universal mechanism for the formation of supported 1D-nanostructures from $\pi$-conjugated molecules that can be combined with other vacuum techniques (e.g., metal sputtering/evaporation, soft plasma etching) for the fabrication of a wide variety of heterostructured nanostructures including hybrid (metal nanoparticles (MNPs)-ONWs) and hierarchical ONWs. ${ }^{[7,8]}$ However, the combination of this
M. Macias-Montero, A. N. Filippin, Dr. F. J. Aparicio, Dr. A. Barranco, Prof. J. P. Espinos, Prof. A. R. Gonzalez-Elipe, Dr. A. Borras

Institute of Materials Science of Sevilla

CSIC - University of Sevilla

Nanotechnology on Surfaces Laboratory

C/Americo Vespucio 49, Sevilla 41092, Spain

E-mail: anaisabel.borras@icmse.csic.es
Dr. Z. Saghi

Department of Materials Science and Metallurgy

University of Cambridge

Pembroke Street, Cambridge CB2 3QZ, UK

Dr. F. Frutos

Applied Physics Department

E.T.S. Ingenieria Informatica

University of Sevilla

Avd. Reina Mercedes s/n, Sevilla 41012, Spain 

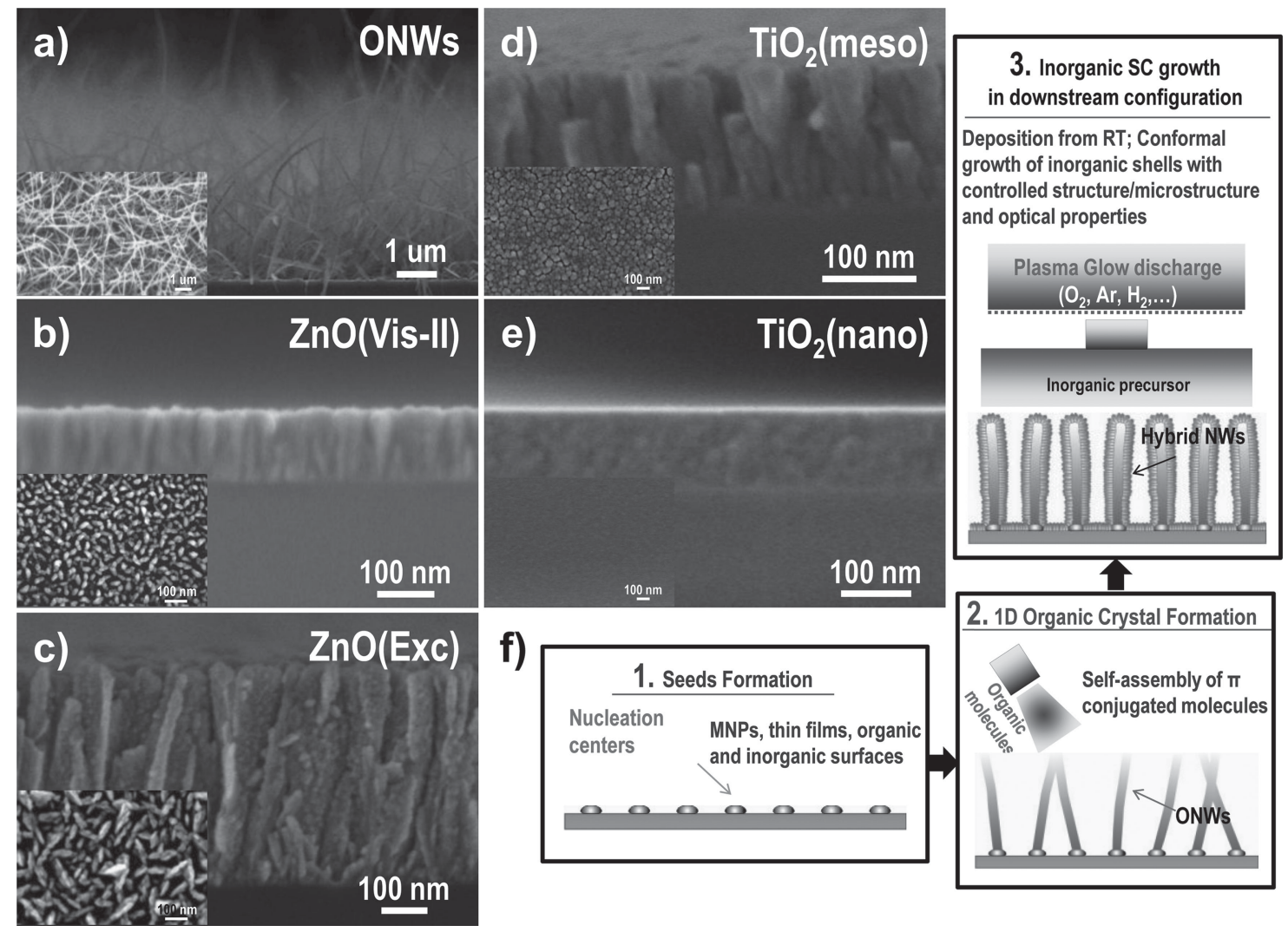

Figure 1. Left: cross section SEM images of a) the as-grown ONWs and b-e) plasma deposited $\mathrm{ZnO}$ and $\mathrm{TiO}_{2}$ thin films deposited on flat substrates as labeled. $\mathrm{ZnO}$ (Vis-II) deposited at room temperature (RT) and $100 \% \mathrm{O}_{2}$ plasma, $\mathrm{ZnO}$ (Exc) deposited at $150{ }^{\circ} \mathrm{C}$ with $10 \% \mathrm{H}_{2} / 90 \% \mathrm{O}_{2}$ plasma, $\mathrm{TiO}_{2}$ (meso) deposited at RT and $100 \% \mathrm{O}_{2}$ plasma, and $\mathrm{TiO}_{2}$ (nano) deposited at $\mathrm{RT}$ and $10 \% \mathrm{O}_{2} / 90 \%$ Ar plasma gas. The insets show normal view SEM images of these films. Right: illustration describing the vacuum methodology used to obtain the supported core/shell organic/inorganic heterostructures: 1) nucleation centers are created on different substrates; 2) a supersaturation regime is reached in the high vacuum chamber and single crystal ONWs formed by self-assembly of $\pi$-conjugated molecules; 3 ) formation of the inorganic shell by PECVD on the as-grown ONWs at low temperature in remote configuration.

method for the formation of 1D organic nanostructures with the synthesis by plasma enhance chemical vapor deposition of inorganic materials has not been explored so far. Thus, this work might suppose the first step in the development of, as it will be seen below, a versatile procedure for the synthesis of heterostructured nanowires.

Therefore, our aim is three-fold: first, to present the synthesis of organic/inorganic core/shell supported NWs by a full-vacuum methodology; secondly, to demonstrate the formation of the inorganic shell with no damage of the organic core single-crystalline structure; and finally, to show the practical application of these organic-core/inorganic-shell nanowires as nano-waveguides. These nanostructures consist of single crystal organic NWs conformally covered by an inorganic shell based on two well-known wide band gap semiconductors, $\mathrm{ZnO}$ and $\mathrm{TiO}_{2}$. In our best knowledge, this is the first example in the literature of such a type of $1 \mathrm{D}$ heterostructure. On the other hand, nanostructured materials based on inorganic semiconductor oxides are commonly used in fields ranging from nanosensors to solar light harvesting, including their outstanding performance as active photonic materials. ${ }^{[9-11]}$ Thus, the third objective is the study of the particular optical properties emerging from the combination of the luminescent ONWs with inorganic transparent nanostructured oxide shells.

The oxides thin films forming the nanowires shells are $\mathrm{ZnO}$ with tunable luminescence, high refractive index $\mathrm{TiO}_{2}$ and low refractive index $\mathrm{SiO}_{2}$. Figure 1 (right) describes the three steps process developed for the formation of these hybrid nanostructures. Step one corresponds to the growth of the nucleation centers for the ONWs formation, for example, MNPs (M: Ag, $\mathrm{Au}, \mathrm{Cu}, \mathrm{Co})$, rough oxide substrates $\left(\mathrm{SiO}_{2}, \mathrm{TiO}_{2}, \mathrm{ITO}, \mathrm{ZnO}\right.$, etc.), ${ }^{[7]}$ or pretreated ONWs. ${ }^{[8]}$ Secondly, a high density array of supported ONWs is obtained by controlling the substrate temperature around 0.5-0.7 times the sublimation temperature of the molecules. In general, the ONWs synthesized by this templateless technique are randomly distributed and bended depicting a spaghetti-like configuration (Figure 1a). In the third step, a conformal inorganic shell is fabricated by plasma enhanced chemical vapor deposition (PECVD), a technique that permits the deposition of thin films at moderate or low temperatures ${ }^{[12,13]}$ (Figure 1b-e). It will be shown that, as a consequence of the inorganic shell deposition, the NWs acquire a vertical configuration which might be optimal for their integration into different top contact electrode devices, still a challenge by the manufacturing of ONWs. ${ }^{[14]}$ Besides the chemical-free, 

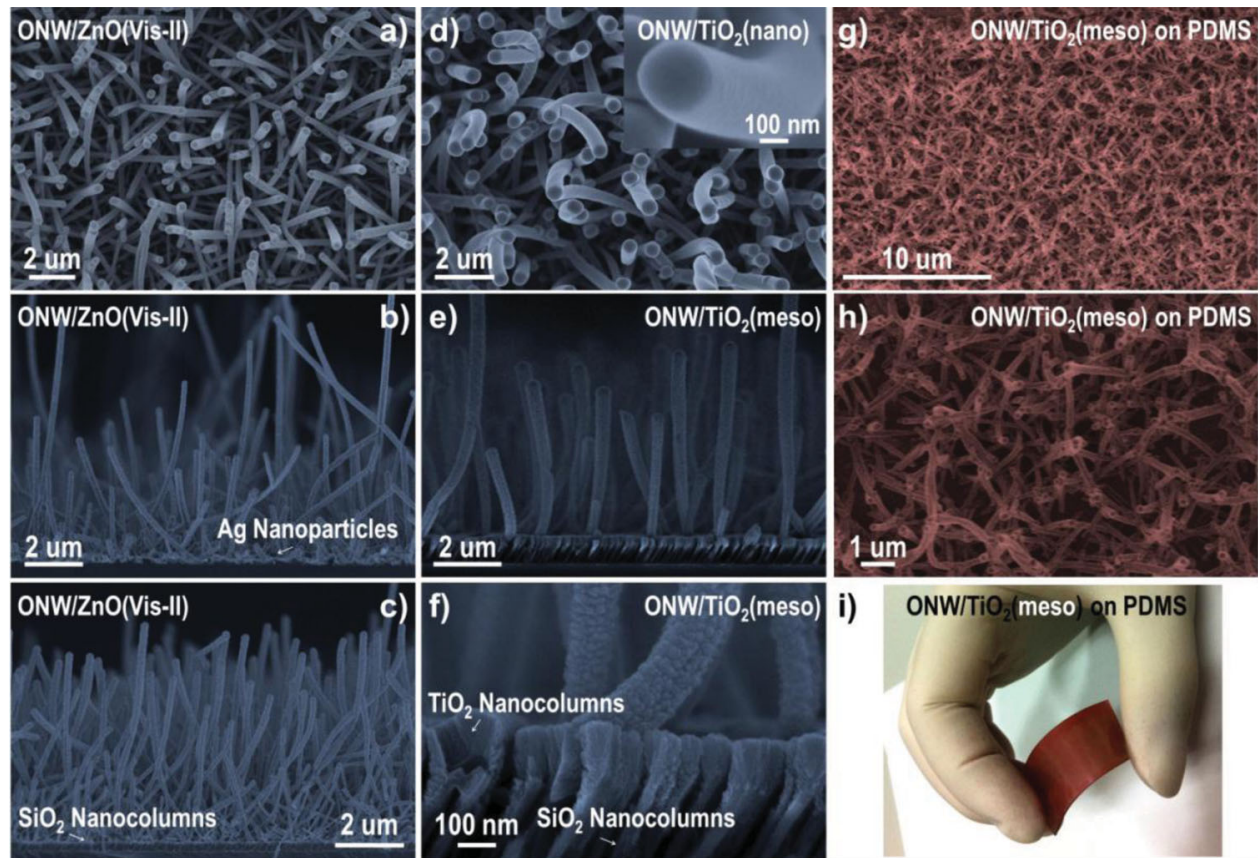

Figure 2. SEM images of the hybrid systems as labeled: $a, b)$ deposited on $\mathrm{Ag} / \mathrm{Si}(100)$ substrates; $\mathrm{c}-\mathrm{f}$ ) on a $\mathrm{SiO}_{2}$ nanocolumnar film; g,h) on PDMS. Color photograph in i) was taken for the hybrid system PtOEP/TiO $($ meso) deposited on the flexible PDMS substrate. This image is characterized by the intense red coloration of the PtOEP NWs.

low energy consuming, and environmental friendly character of the method, it deserves stressing that it is fully convertible to wafer scale and compatible with other techniques applied in microelectronics such as lithography, the use of shadow masks, etching, etc.

\section{Results and Discussion}

\subsection{Fabrication of Supported Hybrid Nanowires: Procedure and Vertical Alignment}

Figure 1a-e gathers selected scanning electron microscopy (SEM) images of the starting materials which are combined for the fabrication of the hybrid NWs including ONWs (Figure 1a) made of different metal phthalocyanines (MPc), metal porphyrins (MOEP) and perylene (MePTCDI), among other smallmolecules. For the shell materials, $\mathrm{ZnO}, \mathrm{TiO}_{2}$, and $\mathrm{SiO}_{2}$ have been deposited by plasma enhanced CVD under different experimental conditions (see Experimental Section) intended for the fabrication of tunable luminescent $(\mathrm{ZnO})$, meso or nano porous $\left(\mathrm{TiO}_{2}\right)$, and low refractive index $\left(\mathrm{SiO}_{2}\right)$ layers. According to previous results, these experimental conditions allow the formation of wurtzite $\mathrm{ZnO}$ nanocolumnar films with a major luminescent emission in the visible for the shells named $\mathrm{ZnO}$ (Vis-I) and $\mathrm{ZnO}$ (Vis-II) and an intense exciton emission around $380 \mathrm{~nm}$ for the $\mathrm{ZnO}$ (Exc) layers. These tunable optical properties are related to the different surface composition, crystal structure, and texture of the thin films deposited on a flat substrate. ${ }^{[12 a]} \mathrm{TiO}_{2}$ amorphous shells of controllable refractive index, $n(550 \mathrm{~nm})$, between 1.80 and 2.20 are provided at the experimental conditions gathered herein. The variable refractive index of the $\mathrm{TiO}_{2}$ is a function of the porosity of the shell, ranging mainly in the mesopores (porous diameter between 2 and $50 \mathrm{~nm}$ ) and in the nanopores (diameter below $2 \mathrm{~nm}$ ) for the $\mathrm{TiO}_{2}$ (meso) and $\mathrm{TiO}_{2}$ (nano) respectively. ${ }^{[12 \mathrm{~b}-\mathrm{c}]}$ SEM images in Figure 1 show the strong difference in microstructure for the inorganic shells from highly facetted crystal columns (Figure 1c) to smooth and continuous layers (Figure 1e). Finally, the $\mathrm{SiO}_{2}$ shell has been included as low refractive index system $(n(550 \mathrm{~nm}) \approx 1.35)$, showing a continuous and nanoporous microstructure. ${ }^{[12 \mathrm{f}]}$ For simplicity, we will name the hybrid structure as $\mathrm{ONW} / \mathrm{ZnO}, \mathrm{ONW} / \mathrm{TiO}_{2}$, or $\mathrm{ONW} / \mathrm{SiO}_{2}$, addressing specifically the composition of the small-molecule and labeling the inorganic shell when required.

Hybrid organic/inorganic core/shell nanowires were fabricated by depositing $\mathrm{ZnO}, \mathrm{TiO}_{2}$, and $\mathrm{SiO}_{2}$ on the as-grown organic nanowires. Figure $2 \mathrm{a}-\mathrm{h}$ shows characteristics SEM images of different hybrid systems deposited on silver NPs (Figure 2a,b), on a $\mathrm{SiO}_{2}$ nanocolumnar thin film (Figure 2c-e), and PDMS (Figure 2g,h). Figure S1 in the Supporting Information gathers the corresponding UV-Vis transmittance spectra of selected hybrid systems on different substrates. Strong absorption peaks in the visible and near infrared range in these spectra are characteristic of the different small-molecule systems. Position of the absorption band-edges and total transmittance identifies substrates and inorganic shells. Figure 2 and Figure S1, Supporting Information, highlight first the general character of the methodology from the point of view of the organic and inorganic counterpart as well as the substrate: metal nanoparticles, inorganic thin film oxides, and flexible organic substrates (cf. Figure 2i) for the photo of a high density 


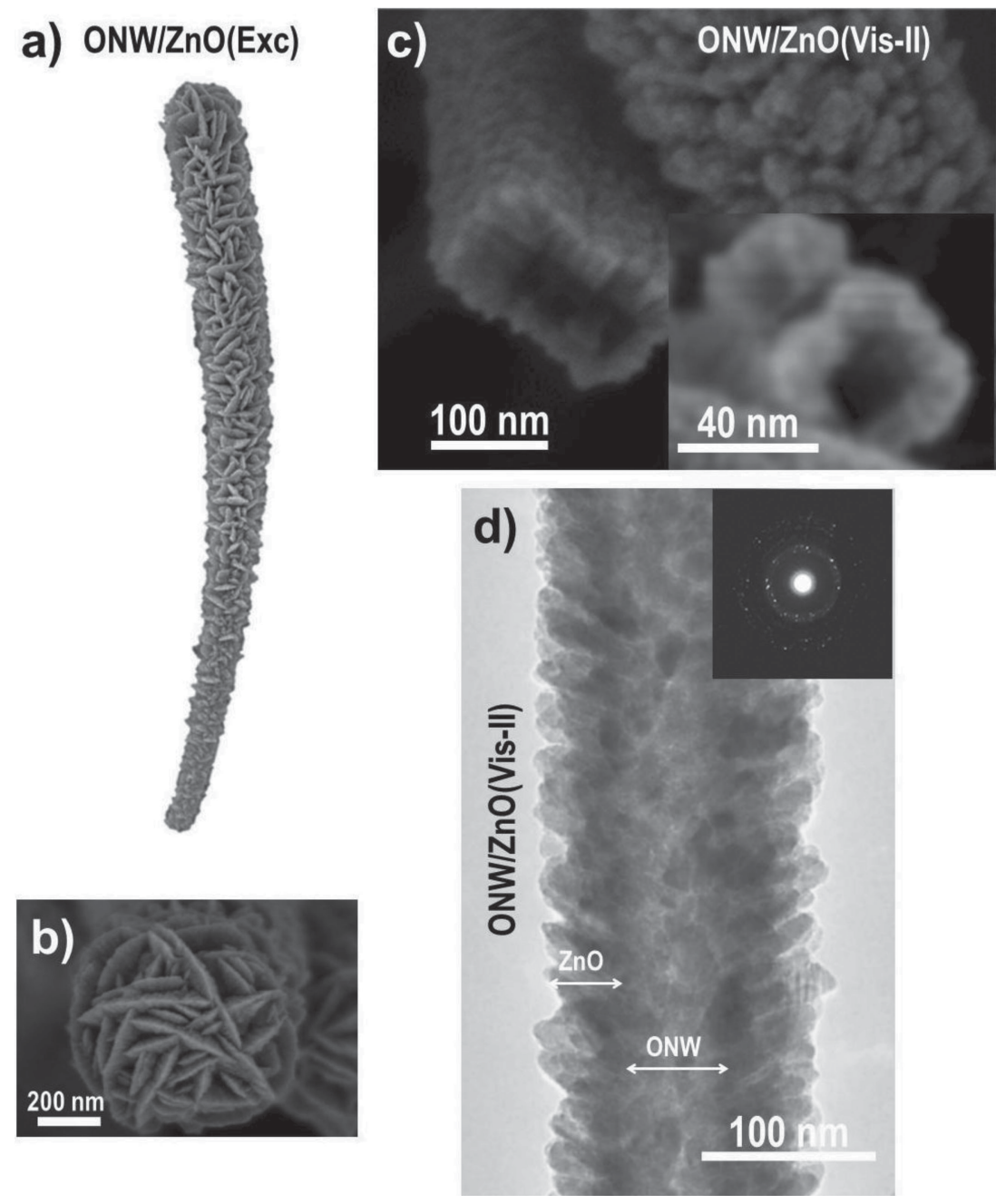

Figure 3. High resolution SEM of $a, b)$ isolated $\mathrm{ONW} / \mathrm{ZnO}(\mathrm{Exc})$ and c) cross-section views of broken hybrid wires; d) transmission electron microscopy (TEM) and selected area electron diffraction (SAED) (inset) of the hybrid NW by bright field mode.

of hybrid nanowires on a PDMS substrate); secondly, the conformal deposition of the inorganic oxides along the ONWs and finally, their vertical alignment. Further confirmation of the second statement is shown in the inset in Figure 2d) and high magnification images in Figure 3a,b, where it is clear that inorganic shell grows conformally around the ONWs forming a smooth coverage in the case of the $\mathrm{TiO}_{2}$ (nano) and a nanocolumnar shell for the $\mathrm{ZnO}$ and $\mathrm{TiO}_{2}$ (meso) conditions. The microstructures of these coating layers are in good agreement with the deposition conditions of the inorganic semiconductors, and resembles those of the films grown on $\mathrm{Si}(100)$ (Figure 1). Thus, $\mathrm{ZnO}$ (Exc) forms highly facetted triangular columns randomly oriented along the ONWs (Figure 3a,b). Meanwhile, the $\mathrm{ZnO}$ (Vis-II) layers yield smoother surfaces and more homogeneous crystal sizes (Figures 2b,c,3d). The $\mathrm{TiO}_{2}$ (nano) deposition conditions lead to the formation of a nanoporous shell characterized by a low roughness surface and continuous coverage (Figure 2d) in contrast with the nanocolumnar $\mathrm{TiO}_{2}$ (meso) growth (Figure 2e,f). Furthermore, the high resolution cross section images of the individual hybrid wires (Figure 3c) show that the conformal inorganic layer grows following the original footprint of the ONW. On other hand, Figure 3a,4 show that, for relatively long deposition times of the inorganic shell, it is produced a preferential accumulation of the coating material at the top of the supported free-standing 1D nanostructures. This theoretically predicted self-shadowing effect ${ }^{[15]}$ does not hamper, in our case, the complete shell conformation of the NWs. In fact, the inorganic layer also covers the free zones of the substrate as clearly evidenced in Figure 2f, even for a high density array of NWs (Figure 2b,c). Additional experiments proved that multi-branched and hierarchical $\mathrm{ONWS}^{[8]}$ can be conformally decorated by PECVD (Figure S2, Supporting Information). It is also clear from Figures 2,4 that the hybrid nanostructures are vertically aligned on the substrate with the tips of the wires pointing upwards after the shell formation. This means the plasma-deposited inorganic coating transforms the randomly oriented and bended distribution of the original ONWs in Figure 1a into a vertical array of hybrid NWs. This effect does not depend on the NW composition and type of substrate, but was enhanced by the thickness of the inorganic shell. Figure 4 gathers characteristic cross-section and normal view SEM images of the formation of $\mathrm{TiO}_{2}$ on organic nanowires for increasing periods of deposition. These images show the incipient vertical alignment of the hybrid wires from the initial stage of the shell formation (Figure 4c,d). The effect is evident for thicker shell thicknesses (Figure 4e,f) and reaches saturation above a certain value (Figure 4g,h). Thus, hybrid wires in the last two panels (Figure $4 \mathrm{~g}, \mathrm{~h}$ ) are characterized by the inverse conical shape related to the selfshadowing effect. The vertical alignment of the hybrid nanowires is more pronounced for low density of ONWs as depicted in Figure 2e, where all the NWs, independently of their length, appear perpendicular to the substrate. Plasma related methods have been thoroughly applied by many authors as a nanofabrication tool of vertically aligned carbon nanotubes (CNTs) and other organic and inorganic 1D nanostructures. Very often, the different reported synthetic strategies takes advantage of the localized electric field in the plasma sheath ${ }^{[13 a-c]}$ that is, generally speaking, normal to the substrate exposed to a low pressure plasma discharge. In the case of the CNTs, ${ }^{[13 b]}$ further control on the orientation can be achieved by application of an external bias, in order to modulate the direction of the electric field. It has been also recently reported the formation of tilted $\mathrm{ZnO}$ nanorods by variation of the geometrical disposition of the substrate during the deposition. ${ }^{[13 \mathrm{~d}]}$ In that case, the growth direction of the $\mathrm{ZnO}$ nanorods was a result of the combination of the plasma sheath electric field and the arrival direction of the $\mathrm{ZnO}$ precursor. We have carried out additional experiments 

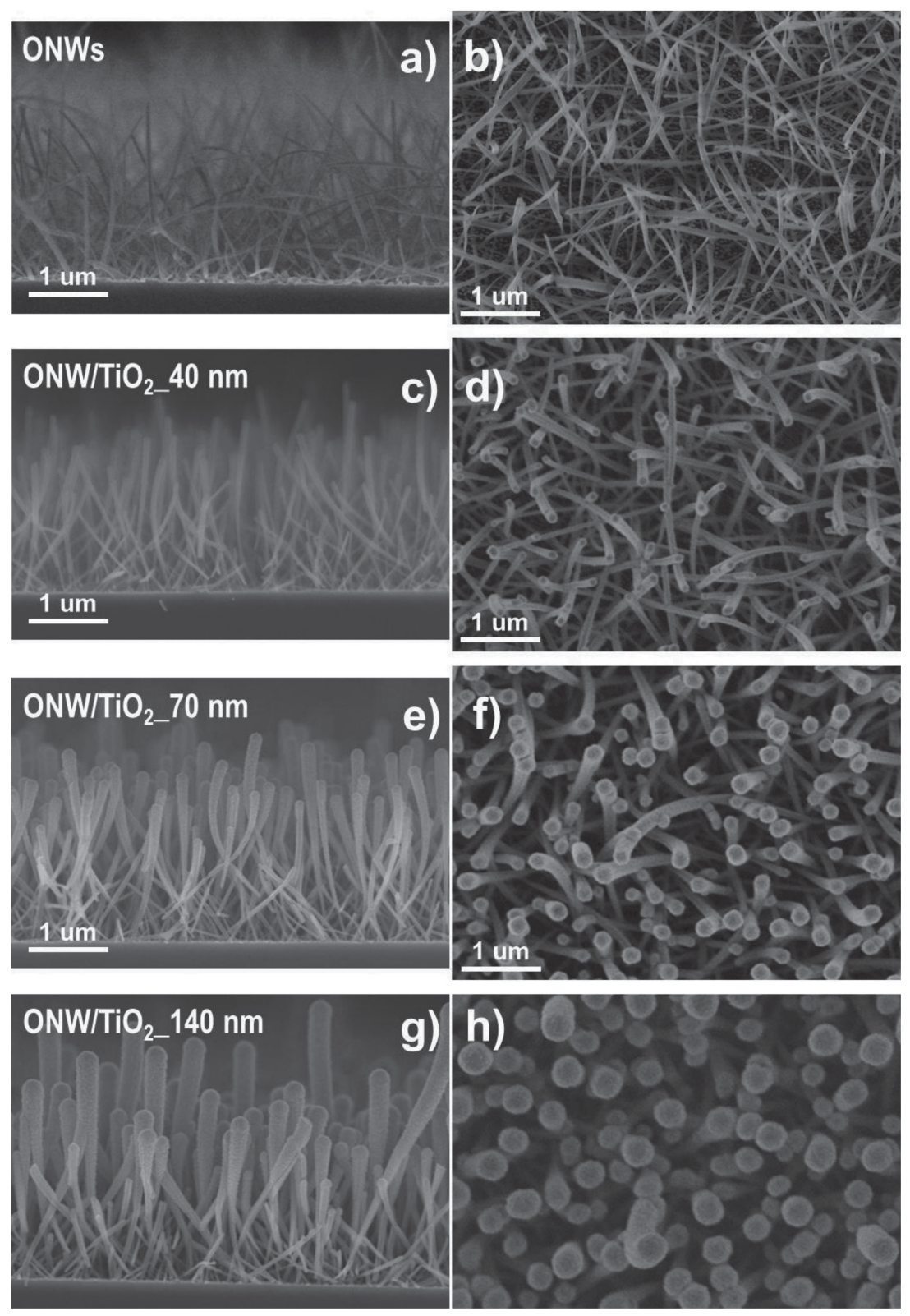

Figure 4. a,c,e,g) Cross-section and b,d,f,h) normal view SEM images of $\mathrm{ONW} / \mathrm{TiO}_{2}$ system fabricated on $\mathrm{Ag} / \mathrm{Si}(100)$ substrates for increasing thicknesses of the $\mathrm{TiO}_{2}$ shell as indicated.

(data not shown) in order to elucidate whether the direction of the precursor flux determines the alignment direction of the hybrid nanowires. Thus, the geometry between the substrates, precursor dispenser, and plasma source was systematically modified. The results of these experiments were always in good concordance with those shown in Figure 4, that is, vertical alignment of the hybrid nanowires along plasma sheath electric field. In addition, the electrostatic force might produce the repulsion between the ONWs that become negatively charge with respect to the plasma and their vertical alignment because the densification of the electric field lines on the tips during the inorganic shell growth. ${ }^{[13 e]}$ Another important factor in our case would also be the increment in the rigidity of the system provided by the formation of the inorganic shell stabilizing the vertical configuration once the plasma discharge is turned off. We foresee that a vertical alignment will prompt the integration of ONWs/ZnO or $/ \mathrm{TiO}_{2}$ nanostructures in different top-contact electrode devices with a wide range of potential application from solar cells to nanosensors.

\subsection{Study of the Crystal Structure of the Hybrid Nanowires: Organic Single Crystal to Inorganic Semiconductor Interface}

For the just mentioned applications, it is essential to prove the semiconducting inorganic shell formation does not damage the ONWs crystal structure and composition. In fact, in a previous publication, we have reported the use of a remote oxygen plasma etching for the controlled fabrication of $1 \mathrm{D}$ composite (ONWs decorated by metal nanoparticles) and hierarchical organic nanostructures. ${ }^{[8]}$ In this process, the plasma etches the wire promoting the arrangement of cations in metallic clusters. It is also well established the role played by oxygen plasma in the formation and stabilization of different inorganic 1D nanostructures, for instance, in the fabrication of metal and metal oxide nanowires by exposition of metal foils to a reactive oxygen plasma. ${ }^{[16]}$ Thus, in the fabrication of the core/shell organic/inorganic nanowires, the effect of ions and other species coming from the plasma discharge might be dramatic for the delicate single crystal configuration of the organic counterpart. However, as it will be demonstrated below, the organic structure remains untouchable and the interface between the organic and inorganic semiconductors is abrupt and well defined. This can be accounted as one of the major achievements of the proposed methodology and deserves an in deep look.

Characterization of the organic crystal structure by electron transmission and diffraction methods is far from being a straightforward task. In fact, the ordered structure of the $\pi$-stacked molecules easily becomes amorphous under the electron beam. ${ }^{[7 b]}$ The high contrast and thickness of the inorganic shells add further handicaps to the full characterization of our hybrid 1D nanostructures. In order to overcome such difficulties, images taken for high resolution transmission electron microscopy (HRTEM) mode in Figure 5 and Figure S3, Supporting Information, were acquired with the samples located at a cryo-holder supplied with liquid nitrogen on broken wires, where the low thickness of the inorganic shell permitted the electron transmission. These images unambiguously show that the parallel line pattern, characteristic of ONWs structures, ${ }^{[5,7]}$ remains unmodified 


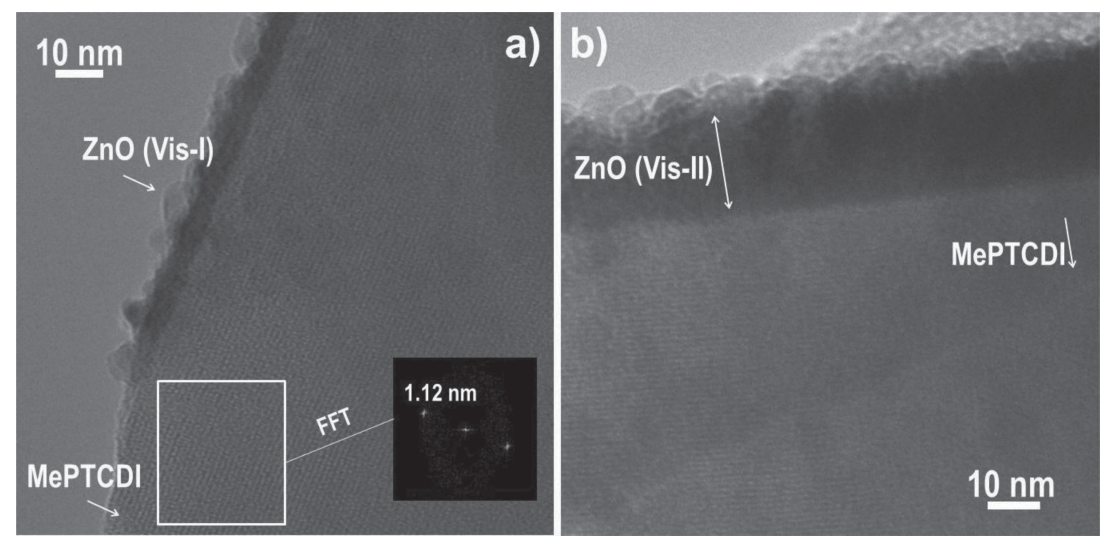

Figure 5. HRTEM images showing that the crystalline arrangement of planes of the organic core remains unaltered after the inorganic component deposition. In both cases, the small molecule forming the ONW was MePTCDI and the shell fabricated in conditions for $\mathrm{ZnO}(\mathrm{Vis})$ at two different thicknesses; inset in a) is the Fast Fourier Transform (FFT) from the selected area.

beneath the ZnO shell. Surprisingly, this characteristic pattern of single crystal ONWs appears unaffected till the interface with the $\mathrm{ZnO}$ layer. This is also true for thicker layers of the inorganic shells (Figure 5b) and even for the most aggressive conditions that we have tested, that is, formation of the $\mathrm{ZnO}$ (Exc) shell at $150{ }^{\circ} \mathrm{C}$ (Figure S3, Supporting Information). The pattern also defines the interline distance (Figure $5 \mathrm{a}$, inset) in this case corresponding to MePTCDI ONWs. ${ }^{[7]}$ These results prove that the formation of the inorganic shell protects the organic structure from oxygen and other reactive species coming from the plasma discharge that might cause damage of the organic crystal or fragmentation of the organic molecules. ${ }^{[8,16]}$ It is worth remarking here that a critical step in the formation of the protective layer is the ignition of the plasma discharge once the metal-organic precursors for the inorganic shell formation have flown into the chamber. We attributed the formation of the well-defined organic-inorganic interface to the effectiveness of the nascent inorganic shell to shield from the start the organic nanowire from the highly reactive ionic and neutral species of the plasma. This aspect clearly deserves future investigations to fully determine the shielding mechanism. Moreover, the use of remote microwave electron cyclotron resonance (ECR) configurations has proven to be a very effective strategy to limit and control the molecular fragmentation of complex molecules interacting with a plasma. ${ }^{[17]}$ For this work, we have deposited the $\mathrm{TiO}_{2}$ shell at room temperature which yields an amorphous layer formation. Substrate temperatures about $250{ }^{\circ} \mathrm{C}$ are required in order to develop the anatase structure. ${ }^{[12 b]} \mathrm{We}$ have succeeded in the formation of ONW/anatase system based in metal-phthalocyanines (data no shown). Unfortunately, the application of this substrate conditions to molecules with lower sublimation temperature such as perylenes has eventually resulted in slimming of the organic core. In the case of the $\mathrm{ZnO}$, the crystal structure is more easily tunable at low temperature by combination of different plasma gases as already cited. ${ }^{[12 a]}$ Structure of the $\mathrm{ZnO}$ shells, once the hybrid structure is formed, has been analyzed by grazing angle X-ray diffraction (GAXRD). Figure S4, in the Supporting Information, gathers the comparison between the hybrid PtOEP/ZnO systems and the reference samples, and demonstrates that the crystal structure of the $\mathrm{ZnO}$ layers on the ONWs is similar to that presented by the $\mathrm{ZnO}$ thin films (see also the selected area electron diffraction (SAED) in Figure 3d). This result is of crucial importance as one of the first examples in the literature of an organic single crystal to semiconducting inorganic crystal interface in a 1D nanostructure. These results can be of significant interest in the design of $\mathrm{p}$ (organic) $-\mathrm{n}$ (inorganic) interfaces when selecting adequately the chemical structure of the organic counterpart.

\subsection{Organic/Inorganic Core/Shell 1D Nano- structures as Optical Waveguides}

Hetero-organic nanostructures have been proposed as white light emitters and waveguide modulators by integrating different organic molecules into binary NWs and nanotubes. ${ }^{[6]}$ Stimulated by these results, we have studied the luminescence properties of the hybrid systems looking for new optical phenomena prompted by the singular luminescent core/shell configuration of the supported nanowires. Luminescence emissions of the hybrid systems comprising MePTCDI as organic NWs are gathered in Figure $6 a-c)$. Figure $6 a$ shows the emission spectra obtained for the $\lambda_{\text {exc }}$ of the organic molecule (i.e., $\lambda_{\text {exc }}=540 \mathrm{~nm}$ ). It is important to address the spectra corresponding to the hybrid systems resembles the one acquired for the reference sample which means that there is no appreciable absorption of light coming out of the organic core by any of the inorganic shells. As shown below, this result allows the use of these structures as nanoscale waveguides in the visible and NIR region. On the other hand, Figure $6 \mathrm{~b}, \mathrm{c}$ presents the luminescence emissions of the MePTCDI/ZnO(Vis-II) and MePTCDI@ZnO(Exc) systems when excited at $280 \mathrm{~nm}$, that is, at the edge of the excitation band for the $\mathrm{ZnO}$ layer. In the spectra of the two $\mathrm{ZnO}$ thin films (Figure 6d) and hybrid samples, the typical near bandedge emission at $\approx 380 \mathrm{~nm}$ and the broad deep level emission of this material in the visible region are neatly observed. The main differences between the emission spectra of ONWs/ZnO and the corresponding to the $\mathrm{ZnO}$ reference thin films are: i) the features at $\approx 550 \mathrm{~nm}$ which can be very likely attributed to partial light absorption from the inner perylene core, and ii) the extended NIR emission due to perylene excitation. The limited absorption of light coming from the emission of the $\mathrm{ZnO}$ shell and consumed in the excitation of the organic nanowire is independent of the composition of the organic molecule (Figure S5, Supporting Information) and, therefore, inherent to the core/ shell configuration. It is also interesting to highlight the ample range of emission depicting by the hybrid systems from the UV to the NIR regions (see, for instance, the broad emission from 400 to $750 \mathrm{~nm}$ in Figure 6b). Besides this singular spectroscopic behavior, the hybrid NWs presented striking light guiding properties as illustrated in Figure $6 \mathrm{e}-\mathrm{i}$. Figure $6 \mathrm{e}$ shows a fluorescence image of an array of MePTCDI NWs supported on quartz. This image depicts a homogenous distribution 

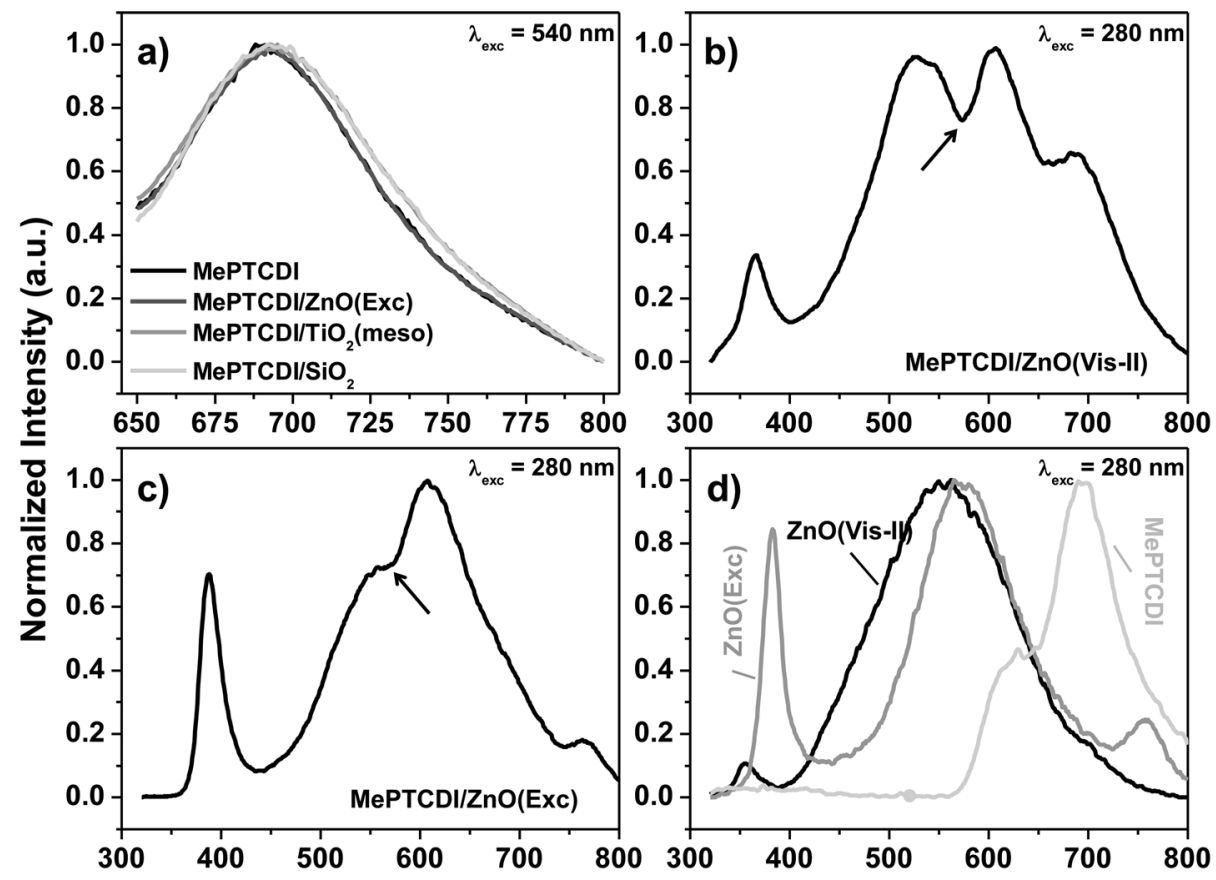

\section{Wavelength $(\mathrm{nm})$}
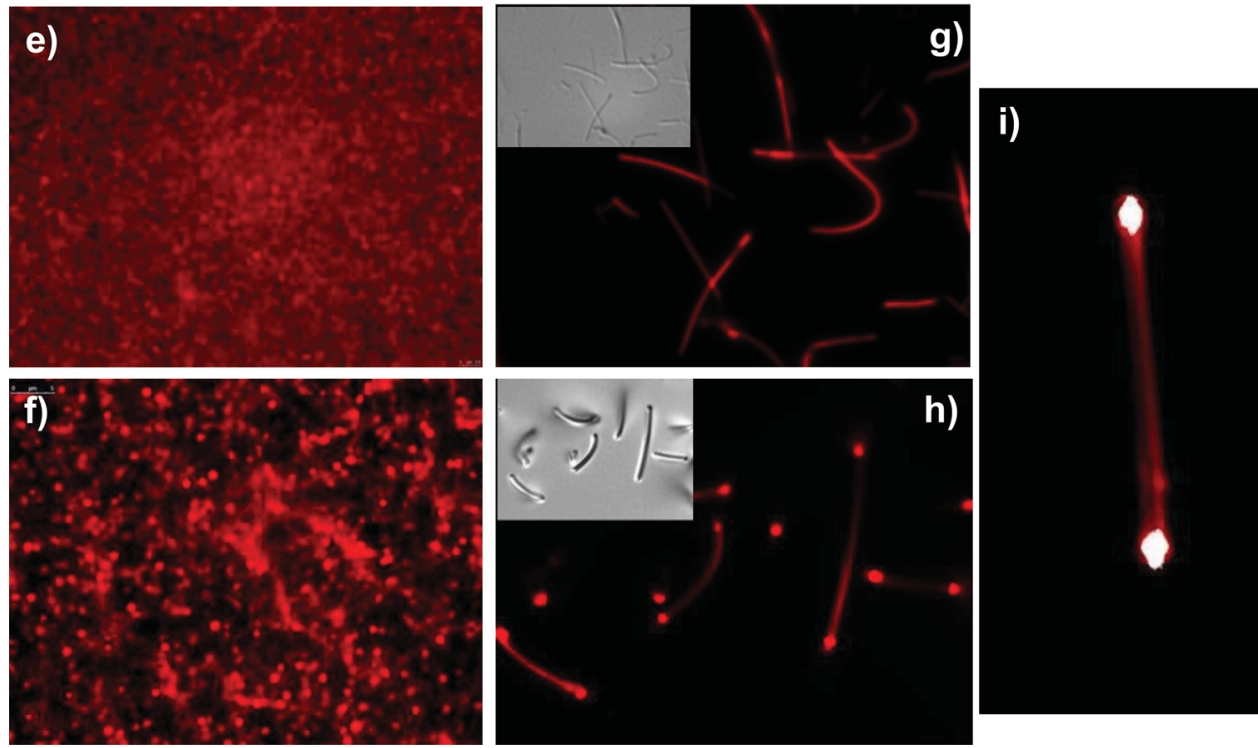

Figure 6. a-c) Normalized photoluminescence spectra of the hybrid systems (MePTCDI/ZnO) as labeled for a) $\lambda_{\text {exc }}=540 \mathrm{~nm}$ and b,c) $280 \mathrm{~nm}$; d) spectra for the reference samples excited at $280 \mathrm{~nm}$. e-g) Fluorescence microscopy images of the organic MePTCDI NWs and f-i) the MePTCDI/ $\mathrm{ZnO}$ (Vis-II) system. These images were recorded by using an excitation wavelength of $550 \mathrm{~nm}$. In the case of panels (e,f), the nanowires were characterized directly on fused silica substrates. Images $(\mathrm{g}-\mathrm{i})$ correspond to nanowires scratched out of the original substrate and emplaced in a microscope slide. The insets in $(\mathrm{g}, \mathrm{h})$ show the equivalent bright field images.

of luminescent structures where the individual nanowires are hardly distinguishable at this level of magnification. The higher magnification image in Figure $6 \mathrm{~g}$ of perylene NWs scratched from the previous surface shows a uniform light emission coming from the entire NW. In contrast, the emission of the MePTCDI/ZnO(Vis-II) supported NWs (Figure 6f) defines a distribution of bright luminescence dots corresponding to the individual supported wire tips. Such an effect is clearly observed in the fluorescence images of the scratched composite NWs in Figure 6h,i. In these examples, the emission of each wire is much more intense in their two tips than in the rest of the wire and, for some of them, only a bright dot in the tip is visible in the fluorescence image (see, for comparison, the bright field image in the inset). This means that the hybrid wires absorb the excitation light and propagate the photoluminescence (PL) emission towards the tips, working as 
a nanoscale waveguide. As expected, the waveguide conditions are a function of the refractive index contrast between the inner organic wire and the inorganic shell. A similar behavior is observed in the MePTCDI/TiO 2 (meso and nano) system with refractive index above 1.85 meanwhile the PL emission for low refractive hybrid core systems as $\mathrm{MePTCDI} / \mathrm{SiO}_{2}$ is analogue to that founded for the original ONWs (Figure S6, Supporting Information).

\section{Conclusions}

We have shown the formation of supported hybrid core/ shell NWs formed by a single crystalline organic core and a conformal inorganic oxide shell. We have demonstrated the ample generality of this methodology from the points of view of the material compositions and type of supports: i) organic nanowire composition, since the PVD of ONWs works effectively for different $\pi$-conjugated small-molecules and the inorganic shell formation is independent of the chemical structure of the ONWs. Thus, the growth by PECVD of the inorganic shell is not constrained by the chemical reaction between the organic molecule and the metal-organic precursor of the shell. In fact, the main restriction for the application of the methodology is the substrates temperature during the plasma activated deposition of the shell. In this article, we have presented the fabrication of wide band gap inorganic semiconductors with no damage of the inner single crystal structure of the ONWs from room temperature to $150{ }^{\circ} \mathrm{C}$. Nevertheless, higher temperatures are sustainable depending, obviously, on the sublimation temperature of the small-molecule forming the ONW. ii) Inorganic shell composition, although we have focused herein in the growth of two key wide band gap semiconductors ( $\mathrm{ZnO}$ and $\mathrm{TiO}_{2}$ ), the formation of the inorganic shell is straightforward applicable to other materials available by PECVD at low and mild temperatures. In fact, results on the formation of ONW/ $\mathrm{SiO}_{2}$ nanostructures have been also included in order to fully describe the optical properties of the hybrid systems. iii) Substrate, both protocols involved in the formation of the organic/ inorganic nanowires are fully compatible with the use of silicon and optical substrates as well as polymeric flexible supports. We have demonstrated the formation of a high density of hybrid nanowires on metal nanoparticles, inorganic thin films and polymers such as PDMS.

It is also shown that, in the course of the plasma deposition of the semiconductor oxide, the NWs acquire a straight configuration likely due to the influence of the electrical field of the plasma sheath. The mild temperature and low plasma power conditions permit the formation of the $\mathrm{ZnO}$ and $\mathrm{TiO}_{2}$ shells with no damaging of the ONWs. Among the wide set of potential applications of this vertically aligned array of hybrid nanostructures, we have examined their performance as waveguide systems at the nanoscale, demonstrating that the fluorescence light produced by the perylene inner core is effectively guided by the semiconducting shell towards the NWs tips. Moreover, we trust exciting results will arise from the generalization of the methodology to other vacuum deposited shells for the fabrication of advanced architectures profiting of the combination of electrically and optically tailored organic and inorganic materials.

\section{Experimental Section}

ONWs Formation by PVD: The PVD procedure for the supported formation of single crystal ONWs has been fully described in previous references. $^{[7,8]}$ Perylenediimide (2,9-dimethyl-anthra[2,1-def:6,5,10-d'e' $\left.{ }^{\prime} f^{\prime}\right]$ diisoquinoline-1,3,8,10-tetrone (Me-PTCDI) from Sensient Imaging Technologies), Pt, and Pd Octaethylporphyrin (Frontier Sci.), and Metal phthalocyanine (Aldrich) were used as purchased (see Scheme S1, Supporting Information, for their molecular structures). ONWs were grown simultaneously on different substrates: $\mathrm{Si}(100)$, fused silica, and $\mathrm{Au}$ TEM grids previously coated with silver nanoparticles by dc-sputtering, and $\mathrm{SiO}_{2}$ and $\mathrm{TiO}_{2}$ thin films deposited by vacuum methodologies. Substrates temperature $\left(T_{\mathrm{S}}\right)$ for the ONWs formation depended on the organic molecule: a) for the Me-PTCDI $T_{\mathrm{S}} \approx 175 \pm 5{ }^{\circ} \mathrm{C}$; b) for the M-OEP $T_{S}$ approximately between 130 to $140{ }^{\circ} \mathrm{C}$; c) for the MPc $T_{S}$ approximately between 240 and $260{ }^{\circ} \mathrm{C}$. These temperatures correspond to the standard working conditions and depend strongly on different experimental parameters such as Argon pressure in the chamber, substrate to Knudsen cell distance, and growth rate of the nanowires.

$\mathrm{ZnO}, \mathrm{TiO}_{2}$ and $\mathrm{SiO}_{2}$ by PECVD: $\mathrm{ZnO}$ and $\mathrm{TiO}_{2}$ thin films were grown onto the ONWs and reference substrates ( $\mathrm{Si}(100)$, fused silica slices, and these substrates previously decorated with silver nanoparticles) by plasma enhanced chemical vapor deposition in a microwave $(2.45 \mathrm{MHz})$ ECR reactor with a down-stream configuration using diethyl zinc ( $\mathrm{ZnEt} 2$ ) as zinc oxide precursor, titanium tetraixopropoxide (TTIP) as titanium dioxide precursor, and chlorotrimethylsilane (CITMS) as silicon oxide precursor. All the precursors were purchase from Sigma Aldrich and used as delivery. ${ }^{[12]}$ The dosed precursor was brought into the chamber by a dosing line equipped with a mass flow controller and sent onto the substrates through a shower-type dispenser located $5 \mathrm{~cm}$ above the sample holder. The dosing line and dispenser were heated about $100{ }^{\circ} \mathrm{C}$, and the mass flow controller at $40^{\circ} \mathrm{C}$, to prevent any condensation in the tube line walls. A thermocouple was used to monitor the temperature of the sample holder that could be heated during the deposition using a halogen lamp. The microwave plasma source (SLAN, Plasma Consult $\mathrm{GMbh}$ ) is coupled to the reaction chamber and separated from it by a grounded grid located $10 \mathrm{~cm}$ above the sample holder and operated at $400 \mathrm{~W}$ for $\mathrm{TiO}_{2}$ and $600 \mathrm{~W}$ for $\mathrm{ZnO}$ and $\mathrm{SiO}_{2}$. Total pressure during deposition was $4 \times 10^{-3}$ Torr for $\mathrm{ZnO}$ and $\mathrm{TiO}_{2}$ and $1 \times 10^{-2}$ for $\mathrm{SiO}_{2}$. Particular experimental conditions for the deposition of the different inorganic shells: i) $\mathrm{ZnO}(\mathrm{Vis}-\mathrm{I})$ and $\mathrm{ZnO}$ (Vis-II) deposited at room temperature (RT) with a $100 \% \mathrm{O}_{2}$ plasma and two nominal thickness of 50 and $100 \mathrm{~nm}$ (Figure 1b); ii) $\mathrm{ZnO}$ (Exc) deposited at $150^{\circ} \mathrm{C}$ with a $10 \% \mathrm{H}_{2} / 90 \% \mathrm{O}_{2}$ plasma and $150 \mathrm{~nm}$ nominal thickness (Figure 1c); iii) $\mathrm{TiO}_{2}$ (meso) deposited at $\mathrm{RT}$ with $100 \% \mathrm{O}_{2}$ plasma and nominal thickness ranging from 40 to $140 \mathrm{~nm}$ and (Figure 1d), iv) $\mathrm{TiO}_{2}$ (nano) fabricated at RT with a $10 \% \mathrm{O}_{2} / 90 \%$ Ar plasma and nominal thickness of $100 \mathrm{~nm}$ (Figure 1e); v) $\mathrm{SiO}_{2}$ fabricated at RT with a $100 \% \mathrm{O}_{2}$ plasma and nominal thickness of $100 \mathrm{~nm}$.

Substrates: Columnar $\mathrm{SiO}_{2}$ thin films were deposited on fused silica slides and $\mathrm{Si}(100)$ by glancing angle vapor deposition as described elsewhere. ${ }^{[18]}$ PDMS preparation is done using the Sylgard 184 Silicone Elastomer Kit from Dow Corning. Silver was deposited by DC sputtering in $\mathrm{Ar}$ at $\approx 0.35$ mbar applying a voltage of $450 \mathrm{~V}$ to a silver thread sited $3 \mathrm{~cm}$ above the substrates. Si (100) wafers, copper grids and fused silica slides were used as substrates. All the substrates were pretreated with plasma before the ONWs deposition in the microwave-ECR remote system. A mixture of gases $\left(25 \% \mathrm{Ar}\right.$ and $\left.75 \% \mathrm{O}_{2}\right)$ was dosed to the chamber whereas the substrates were heated up to $80^{\circ} \mathrm{C}$. This oxygen plasma treatment was carried out for $45 \mathrm{~min}$, at $0.020 \mathrm{mbar}$ of pressure and $240 \mathrm{~W}$ microwave-power.

Characterization: Samples were characterized by SEM as deposited on $\mathrm{Ag} / \mathrm{Si}(100)$ wafers in a $\mathrm{S}-4800$ microscope from Hitachi. For operation in STEM mode, the nanowires were scratched out of the Si(100) over a Lacey TEM grid. GAXRD studies were performed in an X'Pert Pro from Panalytical for $X$-ray angles $<1^{\circ}$. Steady-state luminescence measurements were carried out at room temperature in the front face 
configuration using a Horiba JobinYvon Fluorolog3 fluorimeter equipped with a $450 \mathrm{~W}$ Xe lamp and two monochromators for excitation and one for emission. The excitation monochromators, located between the source and the sample, were used to select the wavelength of the pumping beam. The light emitted by the sample was collected by a photomultiplier (PMT) detector through the emission monochromator. Photoluminescence measurements were performed by scanning the emission wavelength between 300 and $800 \mathrm{~nm}$. The system was equipped with filters in order to remove stray light effects. The measured spectra were corrected from variations of the lamp intensity during the measurements. To compare their luminescence intensities, all the samples deposited were measured under identical experimental conditions. UV-Vis spectra were recorded in a CARY 100 set up for samples handled in air.

\section{Supporting Information}

Supporting Information is available from the Wiley Online Library or from the author.

\section{Acknowledgements}

The authors thank Junta de Andalucia (P09-TEP5283, P09-CTS-5189, and FQM-6900) and the Ministry of Economy and Competitiveness (CONSOLIDER CSD2008-00023, MAT2010-21228, and MAT2010-18447) for financial support.

[1] a) C. N. R. Rao, F. L. Deepak, G. Gundiah, A. Govindaraj, Prog. Solid State Chem. 2003, 3, 5; b) M. Law, L. E. Greene, J. C. Johnson, R. Saykally, P. Yang, Nat. Mater. 2005, 4, 455; c) P. Yang, R. Yan, M. Fardy, Nano Lett. 2010, 10, 1529.

[2] A. J. Mieszawska, R. Jalilian, G. U. Sumanasekera, F. P. Zamborini Small 2007, 3, 722.

[3] a) P. D. Cozzoli, T. Pellegrino, L. Manna, Chem. Soc. Rev. 2006, 35, 1195; b) C. M. Lieber, Z. L. Wang, MRS Bull. 2007, 32, 99.

[4] a) L. Bao, J. Zang, X. Li, Nano Lett. 2011, 11, 1215; b) L. E. Greene, M. Law, B. D. Yuhas, P. Yang, J. Phys. Chem. Lett. 2007, 111, 18451; c) O. Hayden, A. B. Greytak, D. C. Bell, Adv. Mater. 2005, 17, 701; d) A. Walther, J. Yuan, V. Abetz, A. H. E. Müller, Nano Lett. 2009, 9, 2026; e) A. Borras, A. Barranco, A. R. Gonzalez-Elipe, Langmuir 2008, 24, 8021; f) L. F. Cui, Y. Yang, C. M. Hsu, Y. Cui, Nano Lett. 2009, 9, 3370; g) Y. S. Nam, A. P. Magyar, D. Lee, J.-W. Kim, D. S. Yun, H. Park, T. S. Pollom, D. A. Weitz, A. M. Belcher, Nat. Nanotechnol. 2010, 5, 340.

[5] a) A. L. Briseno, S. C. B. Mannsfeld, S. A. Jenekhe, Z. Bao, Y. Xia, Mater. Today 2008, 11, 38; b) L. Zang, Y. Che, J. S. Moore, Acc. Chem. Res. 2008, 41, 1596; c) J. T. Kim, S. K. Seol, J. Pyo, J. S. Lee, J. H. Je, G. Margaritondo, Adv. Mater. 2011, 23, 1968.

[6] a) Q. Liao, H. Fu, J. Yao, Adv. Mater. 2009, 21, 1; b) C. Zhang, Y. S. Zhao, J. Yao, New J. Chem. 2011, 35, 973; c) Y. S. Zhao, H. B. Fu, F. Q. Hu, A. D. Peng, W. S. Yang, J. N. Yao, Adv. Mater. 2008, 20, 79-83; d) R. J. Tseng, J. Huang, J. Ouyang, R. B. Kaner, Y. Yang, Nano Lett. 2005, 5, 1077; e) J. Y. Zheng, Y. Yan, Z. Wang, Y. S. Zhao, J. Huang, J. Yao, J. Am. Chem. Soc. 2012, 134, 2880; f) Y. Zhang, H. Dong, Q. Tang, S. Ferdous, F. Liu, S. C. B. Mannsfeld, W. Hu, A. L. Briseno, J. Am. Chem. Soc. 2010, 132, 11580; g) J. Y. Zheng, Y. Yan, X. Wang, W. Shi, H. Ma, Y. S. Zhao, J. Yao, Adv.
Mater. 2012, 24, OPT194; j) Y. L. Lei, D. Y. Zhou, W. Gu, X. B- Shi, L. S. Liao, S. T. Lee, Adv. Mater. 2012, 24, 5345.

[7] a) A. Borras, M. Aguirre, O. Groening, C. Lopez-Cartes, P. Groening, Chem. Mater. 2008, 20, 7371; b) A. Borras, O. Groening, M. Aguirre, F. Gramm, P. Groening, Langmuir 2010, 26, 5763; c) A. Borras, P. Groening, J. R. Sanchez-Valencia, A. Barranco, J. P. Espinos, A. R. Gonzalez-Elipe, Langmuir 2010, 26, 1487; d) A. Borras, O. Groening, J. Koeble, P. Groening, Adv. Mater. 2009, 21, 4816.

[8] M. Alcaire, J. R. Sanchez-Valencia, F. J. Aparicio, Z. Saghi, J. C. Gonzalez-Gonzalez, A. Barranco, Y. Oulad-Zian, A. R. Gonzalez-Elipe, P. Midgley, J. P. Espinos, P. Groening, A. Borras, Nanoscale 2011, 3, 4554.

[9] a) S. Xu, Y. Qin, C. Xu, Y. Wei, R. Yang, Z. L. Wang, Nat. Nanotechnol. 2010, 5, 366; b) S. H. Ko, D. Lee, H. W. Kang, K. H. Nam, J. Y. Yeo, S. J. Hong, C. P. Grigoropoulos, H. J. Sung, Nano Lett. 2011, 11, 666; c) J. Y. Lao, J. G. Wen, Z. F. Ren, Nano Lett. 2002, 2, 1287.

[10] a) G. K. Mor, K. Shankar, M. Paulose, O. K. Varghese, C. A. Grimes, Nano Lett. 2006, 6, 215; b) U. Diebold, Surf. Sci. Rep. 2003, 48, 53.

[11] a) X. D. Wang, C. Neff, E. Graugnard, Y. Ding, J. S. King, L. A. Pranger, R. Tannenbaum, Z. L. Wang, C. J. Summers, Adv. Mater. 2005, 17, 2103; b) Z. Lin Wang, Mater. Sci. Eng., R 2009, 64, 33.

[12] a) P. Romero-Gomez, J. Toudert, J. R. Sanchez-Valencia, A. Borras, A. Barranco, A. R. Gonzalez-Elipe, J. Phys. Chem. C 2010, 114, 22546; b) A. Borras, J. R. Sanchez-Valencia, J. Garrido-Molinero, A. Barranco, A. R. Gonzalez-Elipe, Microporous Mesoporous Mater. 2009, 118, 314 ; c) A. Borras, R. Alvarez, J. R. Sanchez-Valencia, J. Ferrer, A. R. Gonzalez-Elipe, Microporous Mesoporous Mater. 2012, 160, 1; d) M. Macias-Montero, A. Borras, Z. Saghi, P. Romero-Gomez, J. R. Sanchez-Valencia, J. C. Gonzalez, A. Barranco, P. Midgley, J. Cotrino, A. R. Gonzalez-Elipe, J. Mater. Chem. 2012, 22, 1341 ; e) A. Borras, M. Macias-Montero, P. Romero-Gomez, A. R. Gonzalez-Elipe, J. Phys. D: Appl. Phys. 2011, 44, 174016; f) A. Barranco, J. Cotrino, F. Yubero, T. Girardeau, S. Camelio, A. R. Gonzalez-Elipe, Surf. Coat. Technol. 2004, 180, 244; g) K. Ostrikov, U. Cvelbar, A. B. Murphy, J. Phys. D: Appl. Phys. 2011, 44, 174001.

[13] a) K. Ostrikov, Rev. Modern Phys. 2005, 77, 489; b) M. Meyyappan, L. Delzeit, A. Cassell, D. Hash, Plasma Sources Sci. Technol. 2002, 12, 205; c) C. M. Hsu, C. H. Lin, H. L. Chang, C. T. Kuo, Thin Solid Films 2002, 420, 225; d) M. Macias-Montero, A. Borras, Z. Saghi, J. P. Espinos, A. Barranco, J. Cotrino, A. R. Gonzalez-Elipe Nanotechnology 2012, 23, 255303; e) K. Ostrikov, A. B. Murphy, J. Phys. D 2007, 40, 2223.

[14] Y. S. Zhao, J. Wu, J. Huang, J. Am. Chem. Soc. 2009, 131, 3158.

[15] K. Moore, C. B. Clemons, K. L. Kreider, G. W. Young, J. App. Phys. 2007, 101, 064305.

[16] a) A. Borras, A. Barranco, J. P. Espinos, J. Cotrino, J. P. Holgado, A. R. Gonzalez-Elipe, Plasma Process. Polym. 2007, 4, 503; b) U. Cvelbar, Z. Chen, M. K. Sunkara, M. Mozetic, Small 2008, 4, 1610; c) K. Ostrikov, I. Levchenko, U. Cvelbar, M. Sunkara, M. Mozetic, Nanoscale 2010, 2, 2012; d) M. Meyyappan, M. K. Sunkara, in Inorganic nanowires: applications, properties, and characterization, CRC Press, Inc., Boca Raton, USA 2009.

[17] a) A. Barranco, P. Groening, Langmuir 2006, 22, 6719; b) I. Blaszczyk-Lezak, F. J. Aparicio, A. Borras, A. Barranco, A. Alvarez-Herrero, M. Fernandez-Rodriguez, A. R. Gonzalez-Elipe, J. Phys. Chem. C 2009, 113, 431; c) F. J. Aparicio, M. Holgado, A. Borras, I. Blaszczyk-Lezak, A. Griol, C. A. Barrios, R. Casquel, F. J. Sanza, H. Sohlstrom, M. Antelius, A. R. Gonzalez-Elipe, A. Barranco, Adv. Mater. 2011, 23, 761.

[18] J. R. Sanchez-Valencia, I. Blaszczyk-Lezak, J. P. Espinos, S. Hamad, A. R. Gonzalez-Elipe, A. Barranco, Langmuir 2009, 25, 9140. 